\title{
Hair Growth-Promoting Effect of Resveratrol in Mice, Human Hair Follicles and Dermal Papilla Cells
}

\author{
Yuting Zhang, ${ }^{1}, *$ Chunya $\mathrm{Ni}^{2}, *$ \\ Yan Huang, ',* Yulong Tang,' \\ Kai Yang, ${ }^{2}$ Xiangguang Shi, ${ }^{3}$ \\ Yue Zhang, ${ }^{3}$ Zheng $\mathrm{Li}^{3}{ }^{3}$ \\ ji'an Wang, ${ }^{3}$ Yifei Zhu, ${ }^{3}$ \\ Haiyang $\mathrm{Li}^{3}{ }^{3}$ Yanyun Ma,' \\ Jinran Lin, ${ }^{3}$ Jiucun Wang, (D) 1,3,4 \\ Qingmei Liu, ${ }^{3}$ Wenyu $\mathrm{Wu}^{2,3,5}$
}

'State Key Laboratory of Genetic Engineering, Collaborative Innovation Center for Genetics and Development, School of Life Sciences, Fudan University, Shanghai, People's Republic of China; ${ }^{2}$ Department of Dermatology, Jing'an District Central Hospital, Shanghai, People's Republic of China; ${ }^{3}$ Department of Dermatology, Huashan Hospital, Fudan University, Shanghai, People's Republic of China; ${ }^{4}$ Institute of Rheumatology, Immunology and Allergy, Fudan University, Shanghai, People's Republic of China; ${ }^{5}$ Academy for Engineering and Technology, Fudan University, Shanghai, People's Republic of China

*These authors contributed equally to this work
Correspondence: Qingmei Liu; Wenyu Wu Department of Dermatology, Huashan Hospital, Fudan University, I2 Mid-Wulumuqi Road, Shanghai, 200040, People's Republic of China

Tel +86-2I-52889999

Fax +86-2I-52887782

Email liuqing.mei@huashan.org.cn;

wuwenyu@huashan.org.cn
Background: Oxidative damage has been found in various types of hair loss. As a polyphenolic phytoalexin, resveratrol (RSV) is known as an antioxidant, anti-inflammatory and anti-apoptotic agent.

Objective: Thus, we aim to examine the effects of RSV on hair growth.

Methods: In vivo C57BL/6 mice were used to evaluate the effects of RSV on hair cycle, hair length, skin thickness, hair follicle diameter, hair cycle score and the percentage of hair cycle stage. Then hair shaft length and hair cycle were evaluated by human hair follicles (HFs) ex vivo. The proliferative activities of human dermal papilla cells (hDPCs) cultured in vitro with RSV were assessed using RTCA. The ability of RSV to protect hDPCs against $\mathrm{H}_{2} \mathrm{O}_{2}$-induced oxidative damage is examined by a ROS assay kit.

Results: Topical application of RSV significantly promoted hair growth and stimulated the transition of hair cycle from telogen into the anagen phase on shaved C57BL/6 mice. Ex vivo experiments showed that RSV increased the hair shaft length of HFs and delayed the entry into catagen. In vitro experiments indicated that RSV proliferated hDPCs and prevented hDPCs from oxidative damage caused by $\mathrm{H}_{2} \mathrm{O}_{2}$.

Conclusion: RSV can promote hair growth and may be a potential candidate for the treatment of hair loss.

Keywords: resveratrol, hair growth, hair cycle, hair follicle, dermal papilla cells

\section{Introduction}

Hair loss, or alopecia, is a condition in which hair in some area of the scalp is lost or no longer regrowth. ${ }^{1}$ There are many types of hair loss, such as androgenetic alopecia (AGA) and alopecia areata (AA). Hair loss is caused by genetic factors and external factors especially prominent in modern society, such as work stress, emotional change and hormone secretion disturbance. Its prevalence tends to be among the young and it may result in disturbed self-perception and psychological conflict. Nowadays, it is becoming a worldwide issue for all ages.

Compared to the increasing prevalence of hair loss, approved treatments for hair loss are limited. Currently, minoxidil and finasteride are the only two drugs that have been approved by the United States Food and Drug Administration (FDA) to treat AGA, the most common type of hair loss. ${ }^{2,3}$ Minoxidil is a multifunctional biological response regulator, which contributes to accelerating hair growth, thickening the skin and prolonging the anagen phase of hair cycle. ${ }^{4}$ It is widely used in treating many kinds of hair loss. Another drug, finasteride is a $5 \alpha$-reductase inhibitor, which can reduce the conversion of the androgen testosterone (T) to dihydrotestosterone (DHT), ${ }^{5}$ an excess of which is the main cause of AGA. However, the two drugs have certain limitations. Minoxidil may 


\section{Graphical Abstract}

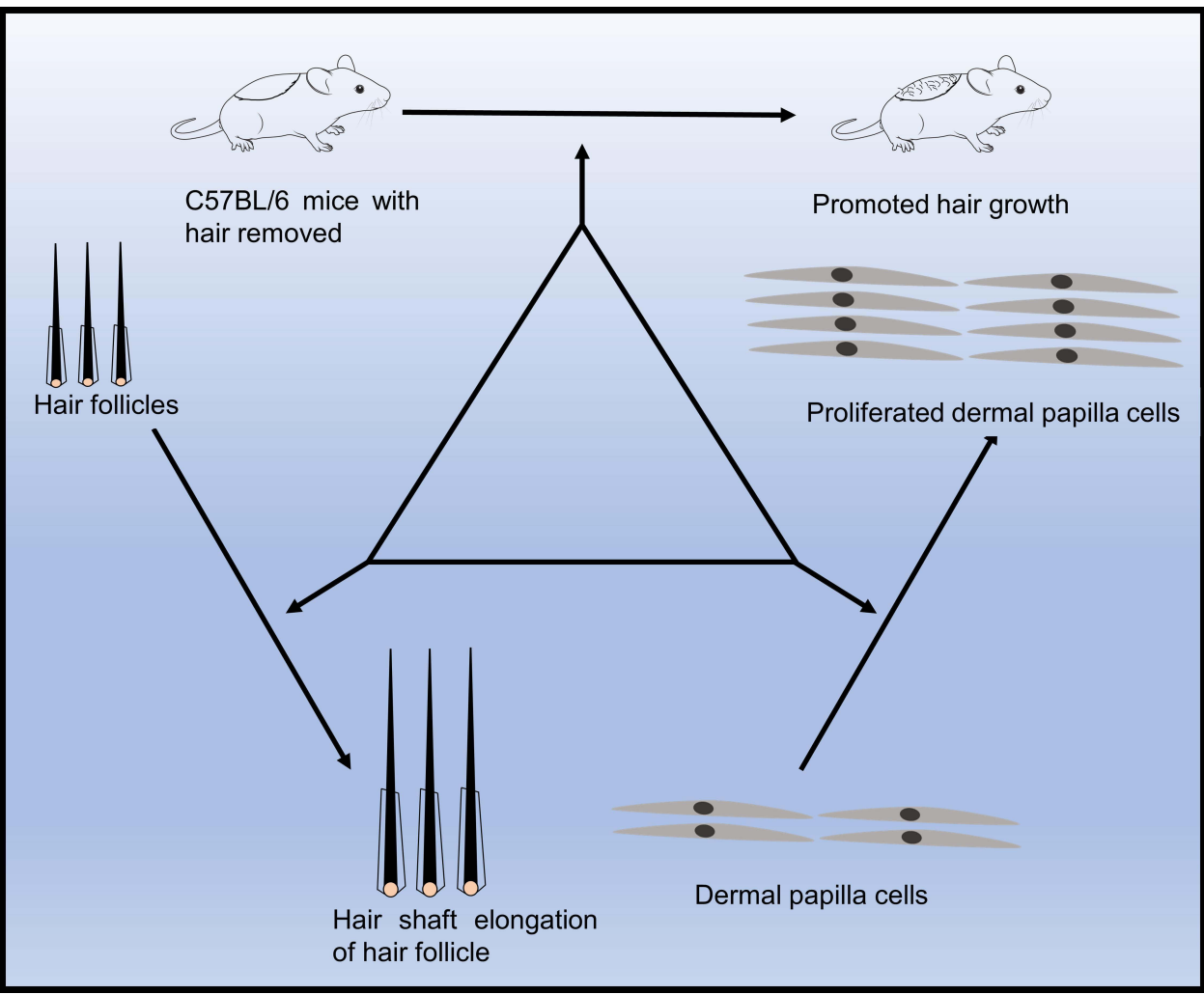

cause pruritus and local irritation while Finasteride has restrictions on use in women. Moreover, they do not work for all patients. ${ }^{6-8}$ Thus, it is necessary to find new substances with fewer side effects.

Hair growth undergoes a cycle of three different phases: anagen, catagen and telogen. In the three phases, anagen is a growth phase of hair shaft, but catagen and telogen are recognized as phases of regression and loss of hair. Longer regression and resting phase and shorter growth phase always occur in hair disorders. Therefore, to help hair growth, it is effective to maintain anagen or shorten catagen and telogen. ${ }^{9}$ The dermal papilla cell (DPC), a mesenchymal derived fibroblast in the hair follicle, play a dominant role in regulating hair cycle and hair growth. It can release a variety of growth factors and transcription factors to help surrounding stem cells differentiate into various structural cells of hair follicles. ${ }^{10,11}$ Decrease of DPC activity, or even apoptosis, can lead to hair loss, including AGA. ${ }^{12}$ Evidence has revealed that some factors increase the level of oxidative damage in hair follicle, leading to the damage of various substances in the cells of hair follicles, which lead to hair loss. ${ }^{13,14}$ Antioxidative capacity of some compounds has been identified as a contributing factor to promote hair growth.

Studies have found that many plant extracts show unique advantages in helping hair growth. ${ }^{15-18}$ Extracts are usually less toxic, less expensive and more available. ${ }^{19}$ Resveratrol (RSV), as a plant polyphenol, was first isolated from the root of Veratrum grandiflorum. It was mainly found in grapes, peanuts, mulberry and other plants. ${ }^{20}$ Previous studies have found that RSV has anti-tumor, antioxidative, antiinflammatory and anti-aging pharmacological effects. ${ }^{21}$ Studies on skin wound healing have also found that RSV can accelerate wound healing, add the number of hair follicles in the skin of young rodents, and promote the production of hair follicles, ${ }^{22}$ but there is a lack of further research. Furthermore, a study found that a combination of RSV with Pyridine-2,4-dicarboxylic acid diethyl ester protects the hair matrix cells in hair follicles from toxic effect of oxidative stress and works well on increasing human hair density, ${ }^{23}$ but it focused mainly on the properties of their combination, not the effect of RSV itself. Therefore, it is speculated that RSV can promote hair growth and may be one of the candidate 
drugs to treat hair loss, but it needs further investigation. In this study, we aim to study RSV's potential in hair growth through in vivo mouse models, ex vivo hair follicle culture models and dermal papilla cells, respectively.

\section{Materials and Methods Experimental Animals}

Six-week-old male C57BL/6 mice were bought and allowed to adapt for 1-2 weeks with food and water ad libitum. To synchronize the mice's hair growth cycle, the hair on their backs was shaved and their backs were treated with liquid rosin under anesthesia on the same day. Then, they were randomly divided into two groups ( $\mathrm{n}=3$ mice/group): RSV group (topical application of RSV dissolved in isopentyl diol at $5 \%$ daily), Control group (topical application of isopentyl diol daily). Pictures of all mice were taken using a digital camera (Panasonic, Japan) on day 0, 7, 14, 18 after depilation. The grayscale of the shaved areas (the region of the back of mice circled by the red line in the photograph) was analyzed by Image $\mathbf{J}$ software to evaluate the process of hair cycle and shown as ratios (grayscale/255). On day 18, new hair on the shaved areas of all mice were plucked to measure their length. The study was reviewed and admitted by the Institutional Animal Care and Use Committee of Fudan University and was performed according to the guidelines of the Science and Technology Commission of Shanghai Municipality (No. SYXK(hu)2020-0011).

\section{Histopathology and Immunofluorescence Analysis}

To further evaluate RSV's effect on hair cycle in vivo, morphological study was conducted on day 5 after treatment because the first few days after shaving are the important time for hair follicles to change from telogen to anagen. ${ }^{24}$ Skin samples resected from the applied site of three mice in every group were fixed in $4 \%$ paraformaldehyde and embedded in paraffin on day 5. The H\&E stained tissues were observed and analyzed through an optical microscope (Nikon, Japan). Skin thickness, which corresponds to hair follicle length, ${ }^{25}$ was defined as the distance between the granular layer of the epidermis and the panniculus carnosus. Each sample was tested in three different areas, and its average value was chosen as every mouse's data. As for HF size, at least 30 hair follicles with visible hair bulb structures were measured and the largest diameter of the hair bulb was selected as the data of per sample. Evaluation of hair cycle per sample were conducted according to Müller-Röver et $\mathrm{al}^{24}$ and
Jing et al. ${ }^{26}$ To be brief, HFs in anagen I-IIIa got a score of 100 , HFs in anagen IIIb-IIIc were 200, and HFs in anagen IVVI were 300. An average score was obtained for each group based on the number of hair follicles. For immunofluorescence staining, the sections were blocked with 5\% BSA (Servicebio, China) and incubated with an antibody to Ki67 (Servicebio, China) overnight at $4{ }^{\circ} \mathrm{C}$. The sections were then incubated with Alexa Fluor ${ }^{\circledR}$ 488-conjugated (Servicebio, China) secondary antibody for $1 \mathrm{~h}$ at room temperature. And TUNEL staining was performed using Fluorescein (FITC) Tunel Cell Apoptosis Detection Kit (Servicebio, China) according to the supplier's recommendations. Cell nuclei were counterstained with 4',6-diamidino-2-phenylindole (DAPI; Servicebio, China). Stained sections were viewed and photographed using fluorescence microscopy (Olympus, Japan).

\section{Human Hair Follicles Organ Treatment}

All hair follicles were obtained from the occipital scalp of hair transplant patients after ethical approval by Huashan Hospital of Fudan University (ethical approval number 2019M-008) and written informed patient consent. The isolated anagen follicles were cultured in $500 \mu \mathrm{L}$ Williams E medium (Gibco, USA) in individual wells of 24 -well plates at $37.0{ }^{\circ} \mathrm{C}$ in $5 \%$ $\mathrm{CO}_{2}$. After $24 \mathrm{~h}$, the HFs that grew $0.3-0.5 \mathrm{~mm}$ were cultured with DMSO or $50 \mu \mathrm{M}$ RSV for 6 days. The picture of hair follicles and the length of hair shaft was obtained with a stereo microscope (Zeiss, Germany). On day 6, each hair follicle was evaluated whether in anagen phase (A) or in catagen $(\mathrm{C})$, and the $\mathrm{A} / \mathrm{C}$ ratio was calculated in each group. The procedure for immunofluorescence staining of $\mathrm{Ki} 67$ and TUNEL is similar to the part of Histopathology and Immunofluorescence Analysis.

\section{Proliferation Analysis of hDPCs}

Human hair dermal papilla cells (hDPCs) were obtained according to the previously described methods. ${ }^{27}$ Cell proliferation was determined by Real Time Cellular Analysis (RTCA) (ACEA Biosciences, USA). A density of 8000 cells per well was seeded in a 16-well microplate. After incubation for $8 \mathrm{~h}$, the medium was removed, followed by the addition of either the medium alone (Control) or the medium with varying concentrations of RSV (30, 35, 40, 50, 60, 125 and $250 \mu \mathrm{M}$, respectively).

\section{Determination of Intracellular ROS of hDPCs}

The intracellular ROS of hDPCs was measured with a ROS assay kit (Sigma, USA) that sets DCFH-DA as 
the probe. There are three groups. One group was treated with $200 \mu \mathrm{M} \mathrm{H}_{2} \mathrm{O}_{2}$ (Sigma-Aldrich, USA) for $12 \mathrm{~h}$ to show the oxidative stage. Another group was treated with $50 \mu \mathrm{M}$ RSV for $24 \mathrm{~h}$ before $\mathrm{H}_{2} \mathrm{O}_{2}$ treatment and control group was treated with vehicles. Then cells were incubated with medium containing $10 \mu \mathrm{M}$ DCFH-DA at $37^{\circ} \mathrm{C}$ for 30 min, and then washed with PBS. The cellular fluorescence intensity was visualized with fluorescence microscope (Olympus, Japan) and the mean fluorescence intensity of each group was measured by Image $\mathrm{J}$ software.

\section{Statistical Analysis}

All the data in the experiments were presented as mean \pm SD. Independent $t$-test and one-way ANOVA were used to perform statistical analyses between different groups, and statistical significance was defined as $\mathrm{P}<0.05$.

\section{Results}

\section{Local Application of RSV on Mice Back Skin Promotes Hair Length and Accelerates Anagen Phase Entry}

To investigate whether RSV has the effect of hair growth, RSV was topically applied to C57BL/6 mice in telogen daily after depilation. On day 0 , the skin at the depilation area of the back of all three groups was pink, indicating their follicles are in telogen phase of hair cycle (Figure 1A). On day 7, all three groups experienced a change in back skin color from pink to gray-black to black (Figure 1A). But the skin color of RSV group is slightly darker than Control group and the corresponding skin grayscale ratio of RSV group is lower than Control group. However, both results have no significant differences (Figure 1B). On day 14, the promoting effect of RSV on the hair of mice started to become obvious. Skin grayscale ratio of RSV group is significantly lower than Control group $(34.06 \% \pm 0.96 \%$ vs $14.89 \% \pm 4.04 \%$, **P $=0.0013$ ) (Figure 1B). On day 18, the back skin of the mice treated with RSV have been mostly filled with hair while dorsal margins of Control group have not been filled with hair (Figure 1A) and the hair length of RSV group was significantly longer compared to the untreated mice $(4.69 \pm 0.35$ vs $9.34 \pm 0.33, * * * * \mathrm{P}<0.0001)$ (Figure 1C). The same results can be seen in Supplementary Figure 1, in which the hair length of RSV group on day 18 was similar to that of positive control group-minoxidil group.

From the histological analysis, it showed that the skin was thicker, the depth of hair follicles was deeper, and the diameter of hair follicles was larger in RSV group than those in Control group (Figure 2B-D) on day 5. It also showed that quite a lot of hair follicles in Control group

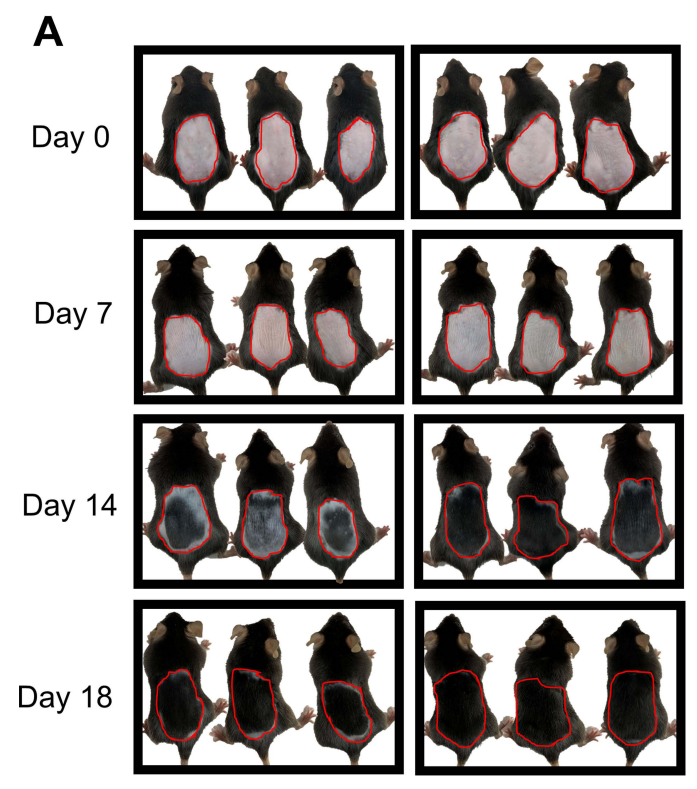

B

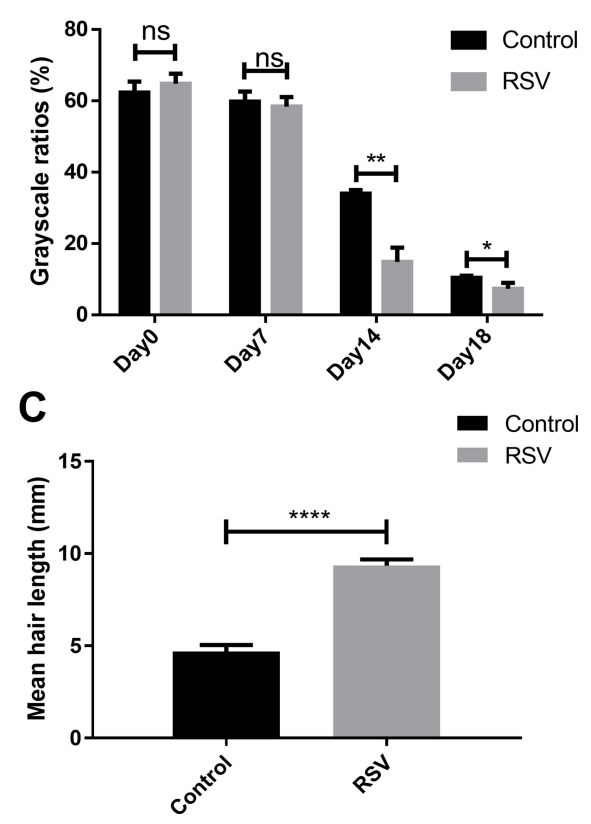

Figure I Macroscopic observations of the hair growth and hair cycle in vivo. (A) Macroscopic pictures of mice on day 0, day 7, day 14 and day 18 . The area circled by the red line is the measurement area of grayscale in (B). (B) The corresponding skin color grayscale ratio on day 0, day 7, day 14 and day 18. (C) Mean hair length on day 18 of each group. Data were presented as mean $\pm S D, n=3$ in each group. The experiments were performed three times with similar results. ns: no significant difference. $* P<0.05$, $* * \mathrm{P}<0.01$, $* * * * \mathrm{P}<0.0001$. 
A

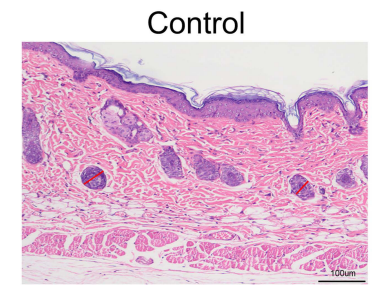

C

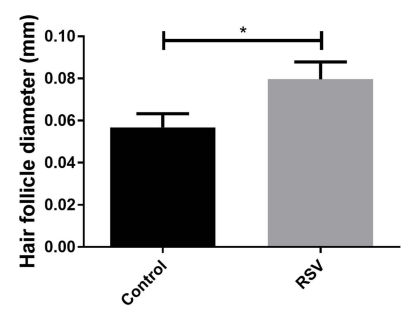

F
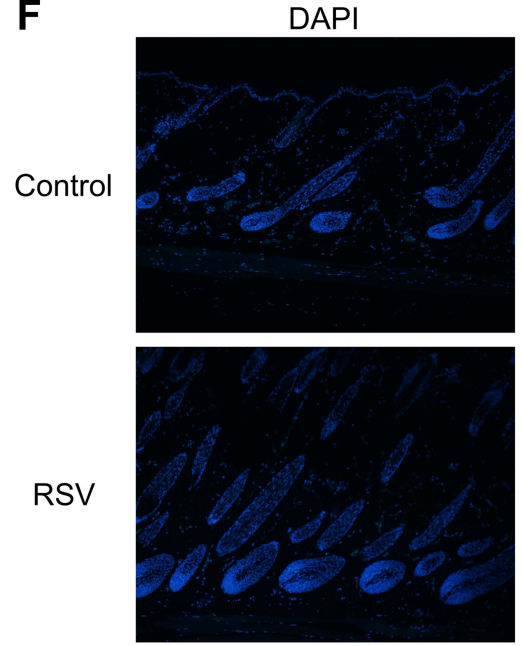

G
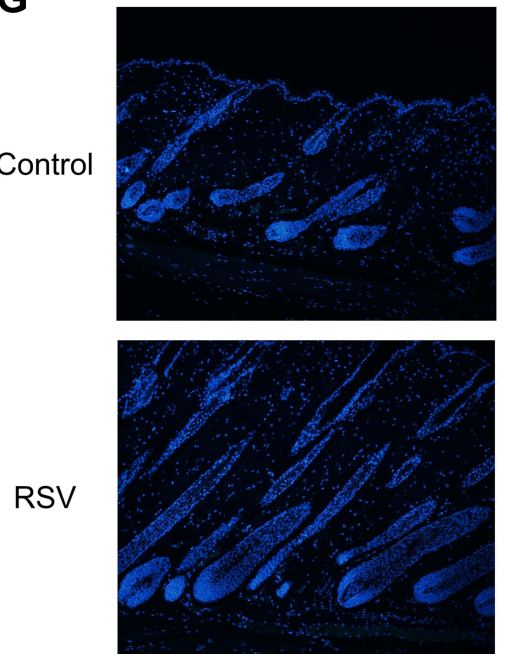

RSV

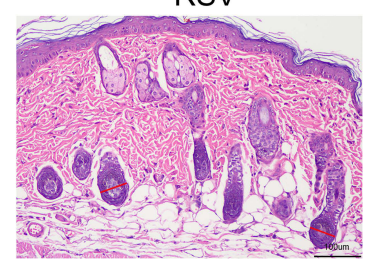

D

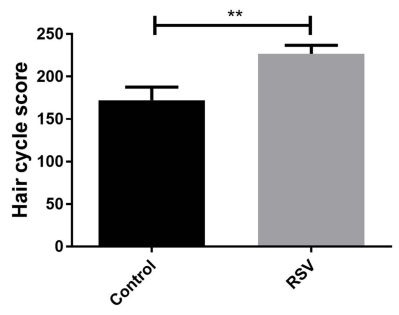

Ki67
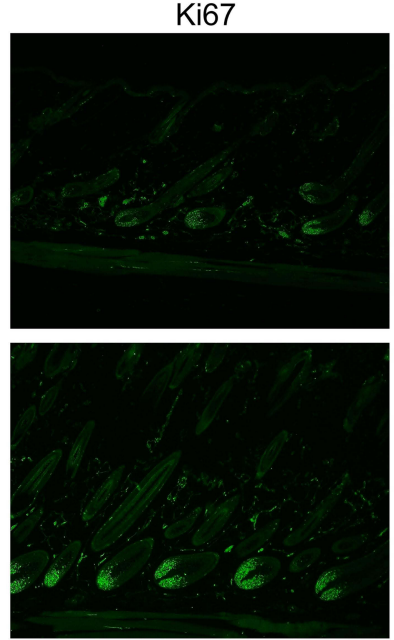

TUNEL
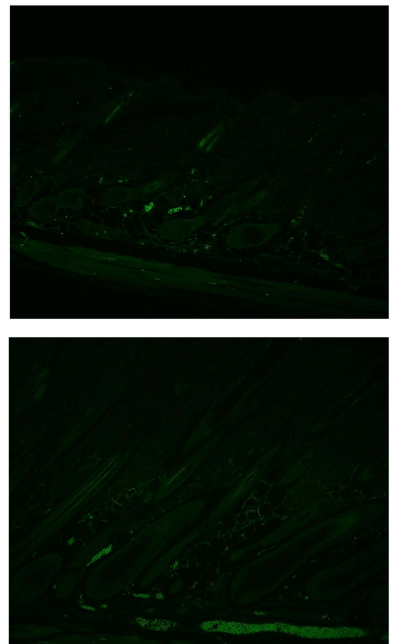

B

E
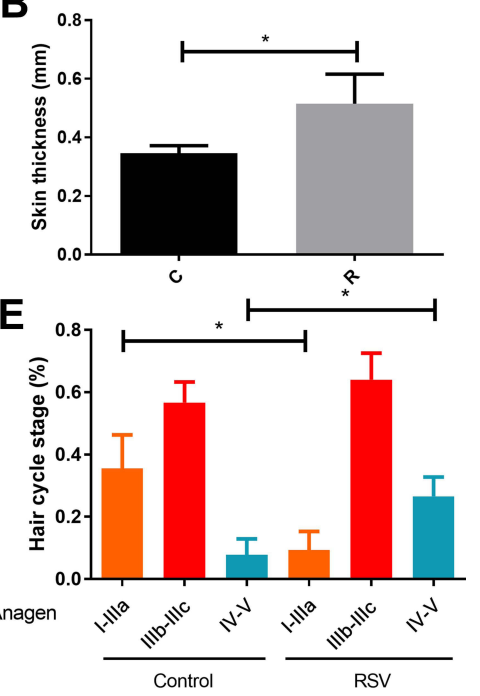

Merge
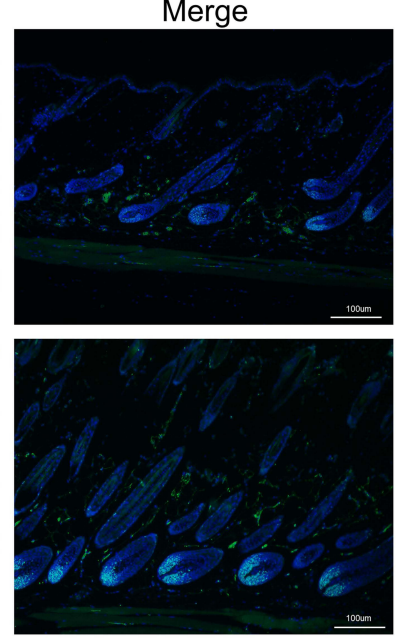

Merge
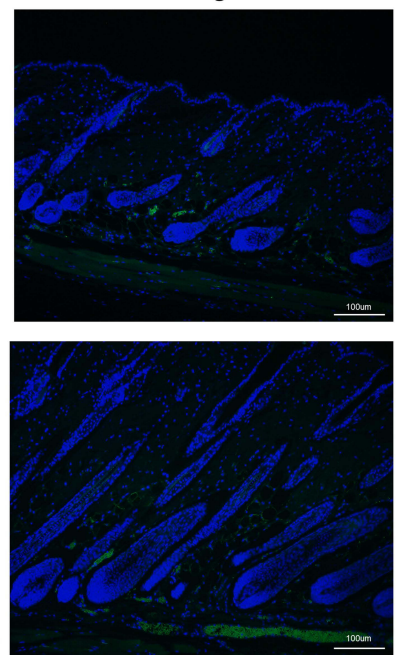

Figure 2 RSV induces anagen entry and development in vivo. (A) A representative histological section of the shaved sites on day 5 after treating with RSV or vehicle. Original magnification was $\times 100$. Red lines indicate the hair bulb diameter. (B) Skin thickness of each group on day 5. (C) The largest hair follicle diameter of each group on day 5. (D) Hair follicles score on day 5. (E) The percentage of three anagen phase. From the left to the right: anagen l-anagen Illa, anagen IIIb-IIlc and anagen IV-VI. (F) Representative image of Ki67 staining on day 7. (G) Representative image of TUNEL staining on day 7. Data were presented as mean \pm SD, $n=3$ in each group. The experiments were performed three times with similar results. $* \mathrm{P}<0.05, * * \mathrm{P}<0.01$. 
were still in the early growth period of anagen phrase (anagen I-anagen IIIa) while more hair follicles in RSV mice entered a later growth period (anagen IV-VI) (Figure 2A and E). On day 7, hair follicles reached the most vigorous stage of growth, at which time proliferating cells (Ki67+cells) increased substantially in RSV group, mainly around hair matrix, outer root sheath and internal root sheath (Figure 2F) while the number of TUNEL+ apoptotic cells in both groups showed no difference (Figure 2G). Taken together, these findings suggested that RSV promotes hair length growth and accelerates anagen entry in vivo by increasing the number of proliferating cells.

\section{Treatment with RSV in Anagen Human HFs Promotes Hair Shaft Growth and Delays Catagen Progression}

HFs ex vivo were used to investigate RSV's effect on the progression of catagen phase, and cultured HFs were divided into two groups (Control, RSV). HFs were taken pictures every other day. The length of hair shaft growth and the hair cycle stage were recorded. The hair shaft of RSV group grew significantly faster than Control group in each passing day (Figure 3A and B). And RSV treatment group went into catagen slower, while Control group entered catagen earlier with a distal retraction of the hair fiber and a reduction in volume of the hair bulb including matrix and hair dermal papilla (Figure 3C and D). On day 6, a larger percentage of HFs remained in anagen phase in RSV group compared to Control group $(77.8 \% \pm 19.2 \%$ vs $19.4 \% \pm$ $17.3 \%, * \mathrm{P}=0.0175$ ), while there was a lower percentage of catagen HFs in RSV group compared to Control group $(22.2 \% \pm 19.2 \%$ vs $80.6 \% \pm 17.3 \%, * \mathrm{P}=0.0175$ Figure 3D). Also, RSV increased Ki67+cells in hair follicles, but had no effect on TUNEL+ apoptotic cells (Figure $3 \mathrm{E}$ and $\mathrm{F}$ ). These results indicate that RSV promotes hair shaft growth and prolongs the anagen of hair follicles in an ex vivo model.

\section{RSV Increased the Proliferation of hDPCs and Protected hDPCs Against Oxidative Stress}

Proliferation of DPCs is essential in hair growth and the process of hair cycle because DPCs have been seen as a key modulator in HFs as described before. In order to assess the effect of RSV on hDPC proliferation, we added RSV at various concentrations to the cultured cells. Lower doses of RSV (30 $\mu \mathrm{M}, 35 \mu \mathrm{M}, 40 \mu \mathrm{M}, 50 \mu \mathrm{M}, 60 \mu \mathrm{M}$ and $125 \mu \mathrm{M})$ accelerated the proliferation of hDPCs (Figure 4A) even though higher dose of RSV $(250 \mu \mathrm{M})$ shows cytotoxicity. Thus, RSV could promote $\mathrm{hDPCs}$ proliferation and the following RSV concentration of ROS experiment was determined as $50 \mu \mathrm{M}$ in this study.

Since RSV is an antioxidant, the ability of RSV to protect hDPCs from the production of ROS in hDPCs was measured by using DCFH-DA as fluorogenic probe. Intracellular ROS was induced by $\mathrm{H}_{2} \mathrm{O}_{2}$. After $12 \mathrm{~h}$ of treatment, $\mathrm{H}_{2} \mathrm{O}_{2}$ treated cells showed a significantly larger number of mean fluorescence intensity compared to control $(32.39 \pm 5.97$ vs $12.92 \pm$ $6.32, * \mathrm{P}=0.0179)$, but the increase in ROS content was prevented if cells were pretreated with RSV for $24 \mathrm{~h}$, as shown by the mean fluorescence intensity of RSV group compared to $\mathrm{H}_{2} \mathrm{O}_{2}$ group $(11.69 \pm 4.40$ vs $32.39 \pm 5.97$, $* * \mathrm{P}=0.0085$ ) (Figure $4 \mathrm{~B}$ and $\mathrm{C}$ ). Thus, these results demonstrate that RSV has a protection for hDPCs against oxidative damage induced by $\mathrm{H}_{2} \mathrm{O}_{2}$.

\section{Discussion}

Although hair loss does not have a major impact on health, it often affects people's appearance, which brings serious psychological burden, and thus affects the quality of life. Therefore, demand for new drugs to treat the condition is growing. RSV is a natural polyphenolic compound which can be found in a variety of plants, including grape berries, and peanuts. Antioxidative and anti-inflammatory properties of RSV imply that RSV may be able to promote hair growth $^{23,28,29}$ because oxidative stress and inflammatory responses are assumed to be involved in the development of hair loss. ${ }^{30-33}$ Therefore, in this study, we used in vivo C57BL/6 mice model, ex vivo human hair follicle cultures and in vitro hDPCs to assess RSV for its hair growth modulating effects.

C57BL/6 mice are a frequently used model in the studies of hair growth and hair cycle. At 6-8 weeks, all hair follicles in C57BL/6 mice enter telogen, and they will no longer enter anagen spontaneously without intervention. ${ }^{24}$ Plucking by liquid rosin or applying depilatory creams can induce their HFs to go back to anagen synchronously, and then go through the three phases of hair cycle (anagen, catagen and telogen) again. ${ }^{34}$ Thus, C57BL/6 mice are an ideal model for the investigation of the functions of RSV on the transition of HFs from telogen to anagen. We found that RSV prompted the transition of hair cycle, which indicates that hair can grow faster with the treatment of RSV in vivo. In 2020, Kubo et al also 

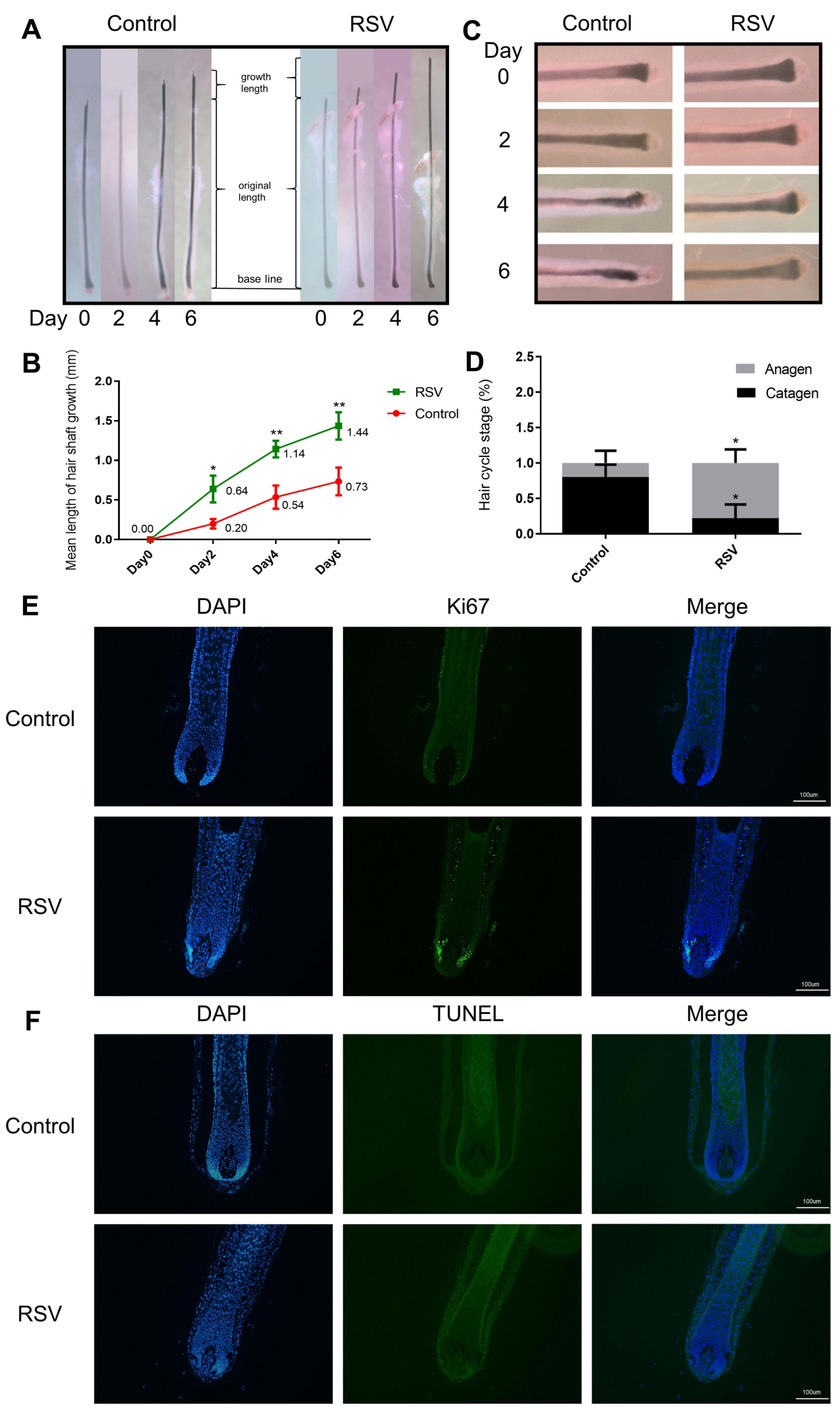

TUNEL
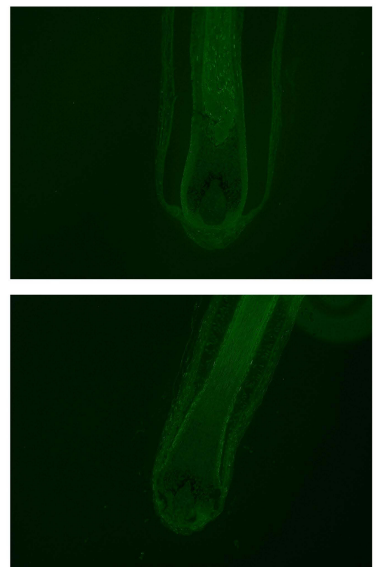

Merge
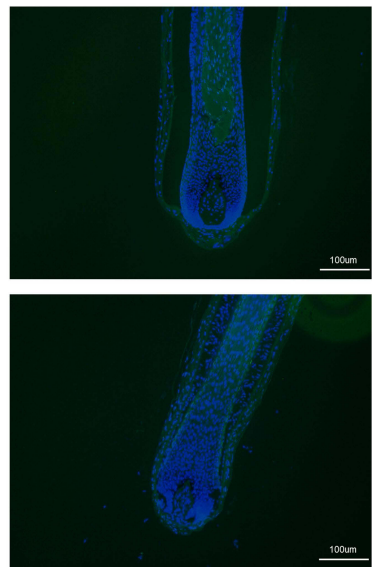

Figure 3 RSV delays catagen entry and increases the length of HFs ex vivo. The HFs were cultured with RSV for 6 days. (A) Photographs of HFs being cultured every other day. (B) Change in hair shaft length of HFs cultured with RSV for 6 days after isolation. (C) Photographs of the bulb in HFs being cultured every other day. (D) Macroscopic quantification of hair cycle stage on day 6 with RSV treatment. (E) Representative image of Ki67 staining on day 2. (F) Representative image of TUNEL staining on day 2. Data are presented as mean $\pm S D, n=3$ in each group. The experiments were performed three times with similar results. ${ }^{*} P<0.05$, $* * P<0.01$. 


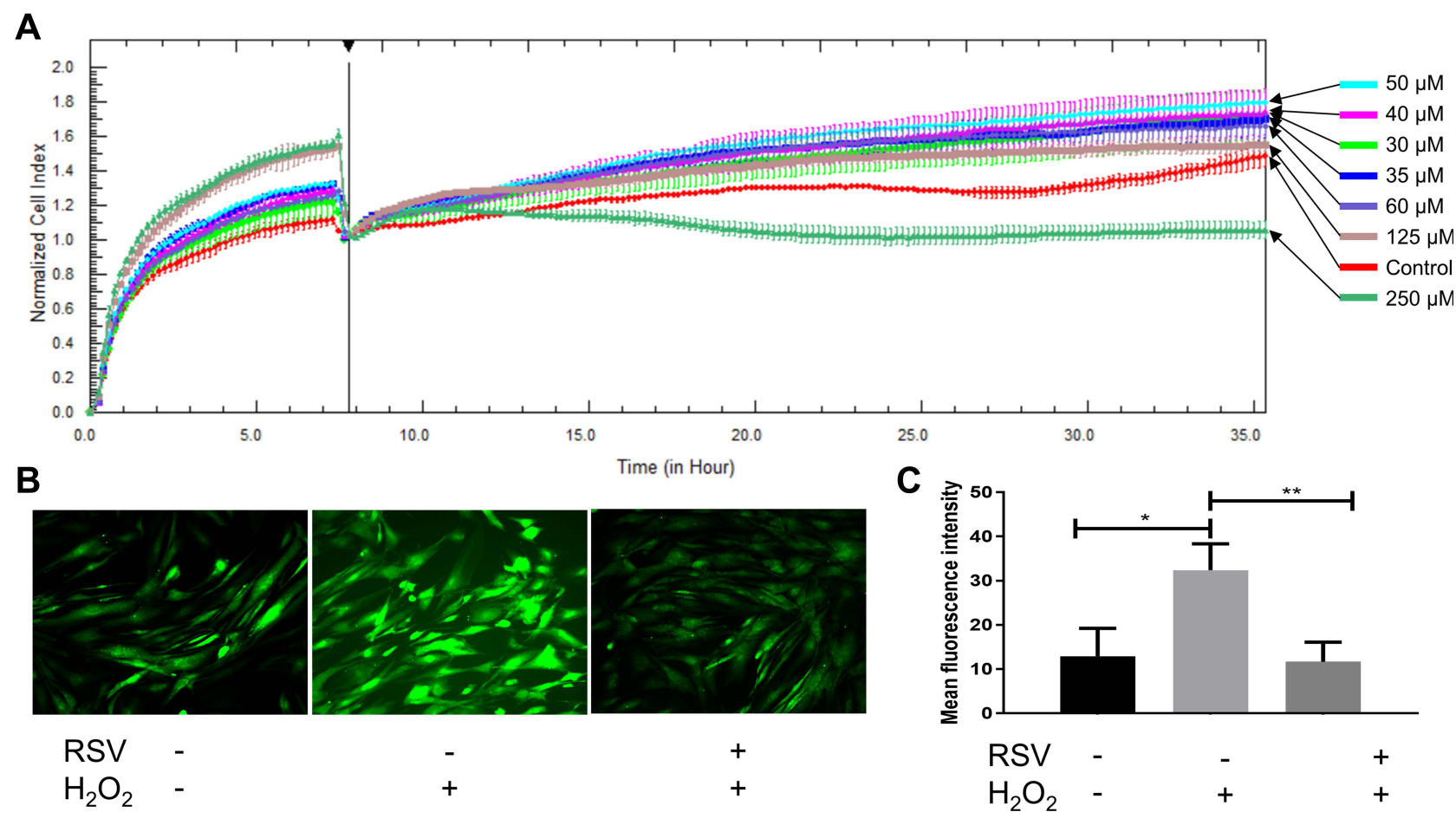

Figure 4 RSV promotes proliferation of cultured hDPCs and protects hDPCs against oxidative stress. (A) Growth curves (measured as cell index over time) of hDPCs treated by RSV with different concentrations. All growth curves were measured with the RTCA system. (B) Photographs taken by fluorescence microscope. (C) Quantification of mean fluorescence intensity of each group. Data are presented as mean $\pm \mathrm{SD}$; $* \mathrm{P}<0.05$; **P $<0.00$ I. The experiments were performed three times with similar results. + , hDPCs were treated with relative materials; -, hDPCs were treated without relative materials.

treated C57BL/6 mice with RSV and found that RSV activated hair growth, although only a small amount of hair grew in the hair removal area. Both of our studies confirmed the effect of RSV on the hair follicles of mice.

HFs are a complex and dynamic mini-organ composed of different anatomical structures, including the dermal papilla, matrix, root sheath, sebaceous gland, and bulge. ${ }^{35}$ Using HFs ex vivo is useful to test the effect of compounds on hair growth as it can largely mimic the state of hair growth in vivo. In this study, we observed that HFs cultured with RSV $(50 \mu \mathrm{M})$ had longer hair shaft and delayed anagen HF's catagen entry, which confirmed the result in animals and it is the first result that evaluated the promoting effect of RSV on HFs ex vivo. All the results indicate that RSV do play a role in hair growth and the cycle of hair follicles in both animals and human organs.

Combined with previous studies, the effect of RSV on hair growth can be in many aspects and in several important cells in hair follicles. First, RSV triggers the proliferation of some important cells in HFs, such as hair matrix cells and DPCs (Figures 1, 2 and 4). Second, RSV protects the cells of hair follicles from environmental damage. We found that RSV prevents hDPCs from oxidative damage by $\mathrm{H}_{2} \mathrm{O}_{2}$. Juchaux et al also found that $\mathrm{RSV}$ can reduce the production of ROS in HFs, especially in hair matrix cells. ${ }^{23}$ Stress induced by environmental factors such as $\mathrm{H}_{2} \mathrm{O}_{2}$, Ultraviolet radiation and DHT will cause damage to DPCs, resulting in hair loss. If hair growth promoters protect DPCs from those stress-related factors, it may suggest that they are effective against hair loss caused by hair follicle damage, such as AGA. Hence, RSV maintains these cells in a better status to help hair follicle growth. DPCs can interact with other cells in hair follicles and are able to release various cytokines to activate several pathways which control the differentiation of the matrix cells and regulate hair growth and development. ${ }^{36}$ With the capability to resist environmental pressure, the matrix cells can also respond more quickly to the regulation of cytokines and signaling pathways and fast differentiate into various structures of the hair follicle, such as outer root sheath cells, internal root sheath cells, keratinocytes.

Among the many signaling pathways that regulate hair growth, Wnt/ $\beta$-catenin pathway plays an important role for the morphogenesis, development, and growth of hair follicles. ${ }^{37,38}$ Kubo et al have found that RSV activated the expression of $\beta$-catenin in both dorsal skin cells of mice and 
HaCaT keratinocytes. ${ }^{28}$ We also found that the mRNA and protein levels of $\beta$-catenin increased in hDPCs after RSV treatment (Supplementary Figure 2). Thus, the activation of $\beta$-catenin might be a key event for hair growth promotion of RSV. Moreover, RSV may accelerate hair growth by accelerating blood flow and enhancing blood supply. Zhao et al found that RSV improved vascularization while promoting wound healing and increased the number of hair follicles. ${ }^{22}$ The increased blood supply allows the hair follicles to better exchange substances, obtaining substances that are good for hair follicle growth and discharging wastes. Therefore, the effect of RSV on hair growth may be carried out on multiple levels, which needs further studies.

Although this study confirmed the promotion effect of resveratrol on hair growth from animal, hair follicle and cell levels, the mechanism of RSV has not been studied in depth and the relationship between various cytokines and multiple pathways is still lacking. In future studies, we aim to clarify the mechanisms underlying RSV-induced regulation of $\mathrm{Wnt} / \beta$-catenin pathway and the interaction between different signaling pathways. Since RSV has the ability to resist environmental stress, it is also a direction of our future research to study the therapeutic effect of RSV on hair loss by using disease models.

\section{Conclusion}

In conclusion, we found that RSV has promoting effects in hair growth and prolongs anagen phase of hair follicle from accelerating anagen entry and delaying catagen progression. Low concentrations of RSV can also mediate the proliferation of DPCs and protect DPCs from oxidative process, but the mechanism of RSV regulating hair follicles needs further studies. Therefore, RSV is expected to be a potential drug for the prevention and treatment of hair loss.

\section{Acknowledgments}

This study was supported by Shanghai Engineering Technology Research Center of Hair Medicine (19DZ2250500), National Science Foundation of China (82173442), Clinical Research Plan of SHDC (SHDC2020CR2033B), CAMS Innovation Fund for Medical Sciences (2019-I2M-5-066), Shanghai Municipal Science and Technology Major Project (2017SHZDZX01), Science and Technology Committee of Shanghai Municipality Guiding Fund (19411962400), and Innovative research team of highlevel local universities in Shanghai.

\section{Disclosure}

The authors report no conflicts of interest in this work.

\section{References}

1. Damodaran RG, Gupta R. Hair loss and the applied techniques for identification of novel hair growth promoters for hair re-growth. Pharmacogn J. 2011;3(22):1-5. doi:10.5530/pj.2011.22.1

2. Lee SW, Juhasz M, Mobasher P, Ekelem C, Mesinkovska NA. A systematic review of topical finasteride in the treatment of androgenetic alopecia in men and women. J Drugs Dermatol. 2018;17(4):457-463.

3. Vañó-Galván $\mathrm{S}$, Camacho $\mathrm{F}$. New treatments for hair loss. Actas Dermosifiliogr. 2017;108(3):221-228. doi:10.1016/j.adengl.2017.02.016

4. Suchonwanit $P$, Thammarucha S, Leerunyakul K. Minoxidil and its use in hair disorders: a review. Drug Des Devel Ther. 2019;13:2777-2786. doi:10.2147/DDDT.S214907

5. Motofei IG, Rowland DL, Tampa M, et al. Finasteride and androgenic alopecia; from therapeutic options to medical implications. J Dermatolog Treat. 2020;31(4):415-421. doi:10.1080/09546634.2019.1595507

6. Kanti V, Messenger A, Dobos G, et al. Evidence-based (S3) guideline for the treatment of androgenetic alopecia in women and in men short version. J Eur Acad Dermatol Venereol. 2018;32(1):11-22. doi:10.1111/jdv.14624

7. Price VH, Roberts JL, Hordinsky M, et al. Lack of efficacy of finasteride in postmenopausal women with androgenetic alopecia. $J$ Am Acad Dermatol. 2000;43(5 Pt 1):768-776. doi:10.1067/mjd.2000.107953

8. Olsen EA, Dunlap FE, Funicella T, et al. A randomized clinical trial of $5 \%$ topical minoxidil versus $2 \%$ topical minoxidil and placebo in the treatment of androgenetic alopecia in men. $J$ Am Acad Dermatol. 2002;47(3):377-385. doi:10.1067/mjd.2002.124088

9. Geyfman M, Plikus MV, Treffeisen E, Andersen B, Paus R. Resting no more: re-defining telogen, the maintenance stage of the hair growth cycle. Biol Rev Camb Philos Soc. 2015;90(4):1179-1196. doi:10.1111/brv.12151

10. Yang CC, Cotsarelis G. Review of hair follicle dermal cells. J Dermatol Sci. 2010;57(1):2-11. doi:10.1016/j.jdermsci.2009.11.005

11. Driskell RR, Clavel C, Rendl M, Watt FM. Hair follicle dermal papilla cells at a glance. $J$ Cell Sci. 2011;124(Pt 8):1179-1182. doi: $10.1242 /$ jcs. 082446

12. Hibberts NA, Howell AF, Randall VA. Balding hair follicle dermal papilla cells contain higher levels of androgen receptors than those from non-balding scalp. $J$ Endocrinol. 1998;156(1):59-65. doi:10.1677/joe.0.1560059

13. Acharya P, Mathur MC. Oxidative stress in alopecia areata: a systematic review and meta-analysis. Int J Dermatol. 2020;59 (4):434-440. doi:10.1111/ijd.14753

14. Prie BE, Voiculescu VM, Ionescu-Bozdog OB, et al. Oxidative stress and alopecia areata. $J$ Med Life. 2015;8(Special Issue):43-46.

15. Murata K, Noguchi K, Kondo M, et al. Promotion of hair growth by Rosmarinus officinalis leaf extract. Phytother Res. 2013;27 (2):212-217. doi:10.1002/ptr.4712

16. Park GH, Park KY, Cho HI, et al. Red ginseng extract promotes the hair growth in cultured human hair follicles. J Med Food. 2015;18 (3):354-362. doi:10.1089/jmf.2013.3031

17. Boisvert WA, Yu M, Choi Y, et al. Hair growth-promoting effect of Geranium sibiricum extract in human dermal papilla cells and C57BL/6 mice. BMC Complement Altern Med. 2017;17(1):109. doi:10.1186/s12906-017-1624-4

18. Kim J, Young Shin J, Choi YH, et al. Hair growth promoting effect of Hottuynia cordata extract in cultured human hair follicle dermal papilla cells. Biol Pharm Bull. 2019;42(10):1665-1673. doi:10.1248/bpb.b19-00254

19. Kaushik R, Gupta D, Yadav R. Alopecia: herbal remedies. Int J Pharm Sci Rev Res. 2011;2(7):1631-1637. 
20. Breuss JM, Atanasov AG, Uhrin P. Resveratrol and its effects on the vascular system. Int J Mol Sci. 2019;20(7):1523. doi:10.3390/ ijms20071523

21. Galiniak S, Aebisher D, Bartusik-Aebisher D. Health benefits of resveratrol administration. Acta Biochim Pol. 2019;66(1):13-21. doi:10.18388/abp.2018_2749

22. Zhao P, Sui BD, Liu N, et al. Anti-aging pharmacology in cutaneous wound healing: effects of metformin, resveratrol, and rapamycin by local application. Aging Cell. 2017;16(5):1083-1093. doi:10.1111/ acel. 12635

23. Juchaux F, Sellathurai T, Perrault V, et al. A combination of pyridine-2, 4-dicarboxylic acid diethyl ester and resveratrol stabilizes hypoxia-inducible factor 1-alpha and improves hair density in female volunteers. Int J Cosmet Sci. 2020;42(2):167-173. doi:10.1111/ ics. 12600

24. Muller-Rover S, Handjiski B, van der Veen C, et al. A comprehensive guide for the accurate classification of murine hair follicles in distinct hair cycle stages. J Invest Dermatol. 2001;117(1):3-15. doi:10.1046/ j.0022-202x.2001.01377.x

25. Andreasen E. Cyclic changes in the skin of the mouse. Acta Pathol Microbiol Scand. 1953;32:157-164. doi:10.1111/j.1699-0463.1953. tb00237.x

26. Jing J, Wu XJ, Li YL, Cai SQ, Zheng M, Lu ZF. Expression of decorin throughout the murine hair follicle cycle: hair cycle dependence and anagen phase prolongation. Exp Dermatol. 2014;23 (7):486-491. doi:10.1111/exd.12441

27. Liu Q, Shi X, Zhang Y, et al. Increased expression of zyxin and its potential function in androgenetic alopecia. Front Cell Dev Biol. 2020;8:582282. doi:10.3389/fcell.2020.582282

28. Kubo C, Ogawa M, Uehara N, Katakura Y. Fisetin promotes hair growth by augmenting TERT expression. Front Cell Dev Biol. 2020;8:566617. doi:10.3389/fcell.2020.566617
29. Rauf A, Imran M, Butt MS, Nadeem M, Peters DG, Mubarak MS. Resveratrol as an anti-cancer agent: a review. Crit Rev Food Sci Nutr. 2018;58(9):1428-1447. doi:10.1080/10408398.2016.1263597

30. Mustafa AI, Khashaba RA, Fawzy E, Baghdady SMA, Rezk SM. Cross talk between oxidative stress and inflammation in alopecia areata. J Cosmet Dermatol. 2021;20(7):2305-2310. doi:10.1111/ jocd. 13814

31. Trueb RM. The impact of oxidative stress on hair. Int J Cosmet Sci. 2015;37(Suppl 2):25-30. doi:10.1111/ics. 12286

32. Simakou T, Butcher JP, Reid S, Henriquez FL. Alopecia areata: a multifactorial autoimmune condition. $J$ Autoimmun. 2019;98:74-85. doi:10.1016/j.jaut.2018.12.001

33. Prie BE, Iosif L, Tivig I, Stoian I, Giurcaneanu C. Oxidative stress in androgenetic alopecia. J Med Life. 2016;9(1):79-83.

34. Stenn KS, Paus R. Controls of hair follicle cycling. Physiol Rev. 2001;81(1):449-494. doi:10.1152/physrev.2001.81.1.449

35. Schneider MR, Schmidt-Ullrich R, Paus R. The hair follicle as a dynamic miniorgan. Curr Biol. 2009;19(3):R132-42. doi:10.1016/ j.cub.2008.12.005

36. Taghiabadi E, Nilforoushzadeh MA, Aghdami N. Maintaining hair inductivity in human dermal papilla cells: a review of effective methods. Skin Pharmacol Physiol. 2020;33(5):280-292. doi:10.1159/000510152

37. Houschyar KS, Borrelli MR, Tapking C, et al. Molecular mechanisms of hair growth and regeneration: current understanding and novel paradigms. Dermatology. 2020;236(4):271-280. doi:10.1159/ 000506155

38. Rishikaysh P, Dev K, Diaz D, Qureshi WM, Filip S, Mokry J. Signaling involved in hair follicle morphogenesis and development. Int J Mol Sci. 2014;15(1):1647-1670. doi:10.3390/ijms15011647

\section{Publish your work in this journal}

Clinical, Cosmetic and Investigational Dermatology is an international, peer-reviewed, open access, online journal that focuses on the latest clinical and experimental research in all aspects of skin disease and cosmetic interventions. This journal is indexed on CAS.
The manuscript management system is completely online and includes a very quick and fair peer-review system, which is all easy to use. Visit http://www.dovepress.com/testimonials.php to read real quotes from published authors. 